\title{
High-throughput analysis of candidate imprinted genes and allele-specific gene expression in the human term placenta
}

\author{
Caroline Daelemans 1,2,3, Matthew E Ritchie4,5, Guillaume Smits'1, Sayeda Abu-Amero2, lan M Sudbery1, \\ Matthew S Forrest1', Susana Campino', Taane G Clark1', Philip Stanier², Dominic Kwiatkowski', Panos Deloukas', \\ Emmanouil T Dermitzakis 1,6, Simon Tavaré4, Gudrun E Moore*+2 and lan Dunham*+1,7
}

\begin{abstract}
Background: Imprinted genes show expression from one parental allele only and are important for development and behaviour. This extreme mode of allelic imbalance has been described for approximately 56 human genes. Imprinting status is often disrupted in cancer and dysmorphic syndromes. More subtle variation of gene expression, that is not parent-of-origin specific, termed 'allele-specific gene expression' (ASE) is more common and may give rise to milder phenotypic differences. Using two allele-specific high-throughput technologies alongside bioinformatics predictions, normal term human placenta was screened to find new imprinted genes and to ascertain the extent of ASE in this tissue.

Results: Twenty-three family trios of placental cDNA, placental genomic DNA (gDNA) and gDNA from both parents were tested for 130 candidate genes with the Sequenom MassArray system. Six genes were found differentially expressed but none imprinted. The Illumina ASE BeadArray platform was then used to test 1536 SNPs in 932 genes. The array was enriched for the human orthologues of 124 mouse candidate genes from bioinformatics predictions and 10 human candidate imprinted genes from EST database mining. After quality control pruning, a total of 261 informative SNPs (214 genes) remained for analysis. Imprinting with maternal expression was demonstrated for the lymphocyte imprinted gene ZNF331 in human placenta. Two potential differentially methylated regions (DMRs) were found in the vicinity of ZNF331. None of the bioinformatically predicted candidates tested showed imprinting except for a skewed allelic expression in a parent-specific manner observed for PHACTR2, a neighbour of the imprinted PLAGL1 gene. ASE was detected for two or more individuals in 39 candidate genes (18\%).

Conclusions: Both Sequenom and Illumina assays were sensitive enough to study imprinting and strong allelic bias. Previous bioinformatics approaches were not predictive of new imprinted genes in the human term placenta. ZNF331 is imprinted in human term placenta and might be a new ubiquitously imprinted gene, part of a primate-specific locus. Demonstration of partial imprinting of PHACTR2 calls for re-evaluation of the allelic pattern of expression for the PHACTR2-PLAGL1 locus. ASE was common in human term placenta.
\end{abstract}

\section{Background}

Although diploid organisms have two copies of each gene, they are not always equally expressed. For some

\footnotetext{
*Correspondence: G.Moore@ich.ucl.ac.uk, dunham@ebi.ac.uk

1 Wellcome Trust Sanger Institute, Hinxton, Cambridge, CB10 1 SA, UK

2 Molecular and Clinical Genetics Unit, Institute of Child Health, London, WC1 $1 \mathrm{EH}, \mathrm{UK}$

† Contributed equally

Full list of author information is available at the end of the article
}

genes, only one allele is active while the other is almost completely silenced. Two different groups of genes fall into this category: genes that exhibit random monoallelic expression, e.g. the odorant receptor genes and genes coding for immunoglobulins [1,2]; and imprinted genes that exhibit monoallelic expression in a parent-of-origin specific manner [3]. Imprinted genes have been shown to be important in fetal and placental development, postnatal growth, behaviour and metabolism [4]. Their regula- 
tion has been found to be disturbed in numerous cancers and dysmorphic syndromes [5].

To date, 56 genes have been identified as imprinted in humans and 98 in mice [6]. A catalogue of human imprinted genes is kept and regularly updated at http:// igc.otago.ac.nz/home.html[7]. However, since most imprinted have been discovered by direct approaches, the total number of imprinted genes is not yet known. Recently, a bioinformatics approach based on DNA sequence characteristics of known imprinted genes predicted 600 imprinted genes in mice [8]. In the human, statistical models have been developed to identify genes with unequal representation of alternative alleles in the public EST libraries, suggesting a further 55 candidate imprinted genes [9]. Many imprinted genes are expressed in a parent-of-origin specific manner in the placenta, making it a "first choice" tissue in which to screen for new imprinted genes [10].

Imprinted expression is at the extreme end of the autosomal allelic imbalance spectrum. However, more subtle allelic variations around the expected 50:50 ratio of expression have been documented. Yan et al. were the first to report such ASE in human [11]. They studied 13 genes and detected 1.3 to 4.3-fold expression differences between alleles for six of them. Lo et al. studied 1063 genes (using Affymetrix HuSNP array) in seven fetuses, where of the 602 genes that were heterozygous, 326 showed preferential expression of one allele in at least one individual (54\%), while 170 (28\%) showed more than a four-fold difference between the two alleles [12]. Several oligonucleotide microarrays have been used to study ASE in lymphoblastoid cell lines (LCLs). Pant et al. used a custom made microarray (Perlegen, USA) and found allelic expression differences in at least one individual in $53 \%$ of the 1389 genes targeted by heterozygous single nucleotide polymorphisms (SNPs) [13]. More recently, Gimelbrant et al. found monoallelic expression for $7.3 \%$ of the genes they tested in clonal lymphoblastoid cells [14]. Strong ASE differences (ASE ratio $>4$ or $<1 / 4$ ) have been found by Bjornsson et al. in 10\% of SNPs in LCLs [15]. Hence, it seems that ASE is frequent, possibly underlying much of human variability [11-15].

We have screened human term placenta for novel imprinted genes and ASE using two technologies that have been shown to be able to quantify allelic expression in a medium and high-throughput manner: the MassArray system (Sequenom, Inc.) [16] and the Illumina ASE Bead Array $^{\mathrm{TM}}$ [17], respectively.

\section{Results}

\section{Sequenom}

The MassArray system (Sequenom, Inc.) was used to test 143 genes for ASE in at least 23 family-trios. Each trio consisted of placental genomic DNA (gDNA), placental cDNA and both parental gDNAs. We analysed six imprinted control genes, seven biallelically expressed genes, seven orthologues of mouse imprinted genes, 99 orthologues of mouse imprinted candidate genes [8], and 26 human imprinted candidate genes [9] (Additional file 1: Supplemental Table S1). For 123 genes (86\%), the cDNA amplification was successful and at least two placentas were heterozygous. A $t$-test (followed by FDRmoderation) was used to verify the null hypothesis that there was no allelic imbalance between the ratios of alleles in gDNA and in cDNA (Table 1 and Methods).

Five imprinted control genes exhibited imprinting (no informative sample for rs2066707-ATP10A). In the subset of genes with acceptable cDNA genotyping success (arbitrarily set at a ratio between cDNA and gDNA genotyping higher than $75 \%$, see Methods), six candidate genes were significant for allelic imbalance in cDNA $(\mathrm{p}<0.05)$ (Table 1). None of these genes had an allelic expression pattern that was compatible with imprinting. Of these, RASGRF1 had the most allelic difference (76\%) and it is notable that the mouse orthologue Rasgrf1 is imprinted in the brain [18]. Its mode of allelic expression in human term placenta was compatible with random monoallelic expression (no allelic preference; four paternal, one maternal and three biallelic mode of expression; data not shown). We checked the mode of expression of RASGFR1 in the human term placenta by Sanger sequencing. Biallelic expression (with sometimes a very slight random bias between alleles) was found in seven informative term placenta samples (data not shown). The average fluorescence level of RASGFR1 on the Illumina array was below our cut-off suggesting low expression level (see below). We thus considered RASGRF1 random monoallelic ASE to be a false positive.

Using rs4911163 as a readout, ACSS2 showed a statistically significant (two-tailed $t$-test, $\mathrm{p}=0.0075$ ) preferential mode of ASE (Additional file 2). Using the Genevar database (T-P. Yang and E. Dermitzakis, manuscript in preparation), variable level of expression for ACSS2 in relation to rs4911163 genotype was also found in lymphoblastoid cells of HapMap3 individuals (B. Stranger and E. Dermitzakis, manuscript in preparation; $[19,20])$. ACSS2 is a cytosolic enzyme that catalyzes the activation of acetate for use in lipid synthesis and energy generation. It has no known function in relation to placenta.

The four other genes presented a much less convincing ASE pattern and were probably false positives. Three of them (DISC1, C9orf93, TF) were present on the Illumina array (see below) and had low expression levels (average $\log _{2}$ fluorescence lower than 11.25). In conclusion, the Sequenom platform can detect ASE and imprinting, but no new imprinted gene was found in this study. 
Table 1: SNPs and corresponding genes statistically significant when tested for ASE by Sequenom Assay.

\begin{tabular}{|c|c|c|c|c|c|}
\hline Gene & SNP_ID & fdr.p.values & Difference & SR ratio & Mode of ASE \\
\hline DLK1 & rs1802710 & $3.62 \mathrm{E}-23$ & 88.6 & 0.89 & Imprinting \\
\hline PEG3 & rs1860565 & $2.74 \mathrm{E}-22$ & 98.1 & 1.00 & Imprinting \\
\hline IGF2 & rs680 & $2.32 \mathrm{E}-16$ & 94.6 & 1.04 & Imprinting \\
\hline PEG10 & rs13073 & 4.19E-08 & 98.4 & 0.84 & Imprinting \\
\hline PHLDA2 & rs13390 & 4.19E-08 & 98.1 & 1.13 & Imprinting \\
\hline DISC1 & rs821616 & 0.022009568 & 15.3 & 0.91 & Random ASE† \\
\hline RASGRF1 & rs11855231 & 0.022009568 & 75.7 & 0.95 & Random monot \\
\hline C9orf93 & rs1539172 & 0.039790941 & 30.0 & 0.78 & Preferentialt \\
\hline$T F$ & rs8649 & 0.04122505 & 56.9 & 0.78 & Random ASE $†$ \\
\hline ACSS2 & rs4911163 & 0.04122505 & 21.9 & 0.86 & Preferential \\
\hline KIAA0523 & rs3744725 & 0.04122505 & 36.5 & 0.96 & Random ASE† \\
\hline
\end{tabular}

The $p$-value is adjusted for multiple testing (false discovery rate bound). The average difference of expression between the two alleles in the CDNA of heterozygous individuals is greatest for imprinted genes. SR ratio is the ratio of genotyping success rate of cDNA on gDNA. Mode of ASE summarises the pattern of ASE based on the quantitative allelic expression data. †False positive pattern probably due to a low expression level (see text for details).

\section{ASE Illumina Array}

To test more candidate genes, we increased our screening throughput by using the ASE BeadArray ${ }^{\text {TM }}$ (Illumina, Inc., USA). With this technique a total of 1536 SNPs, located in 932 genes (214 expected to be expressed in placenta, see Methods) (Additional file 1: Supplemental Table S2), were tested for ASE and imprinting across 23 of the family-trios. The candidate imprinted genes included ten orthologues of known murine imprinted genes whose status was unknown in human, 124 orthologues of 600 mouse candidate imprinted genes [8], ten human candidate imprinted genes [9], and 18 known control imprinted genes $[6,13,21]$ (Additional file 1: Supplemental Table S2). Genes specifically expressed in the placenta compared to other tissues and genes differentially expressed according to the birth weight may influence fetal growth and so may also be imprinted. We therefore tested 46 such genes [22]. The remaining 1179 SNPs (718 genes) on the array were chosen for unrelated research purposes and were thus randomly selected in terms of this study. This study also duplicated 38 genes from the Sequenom analysis on the same samples.

\section{Comparison of platforms}

For comparison, we analysed the results obtained for the 38 genes tested on both platforms for the same familytrios (Figure 1). These results were used to determine the minimum cDNA intensity necessary for the Illumina platform to correlate for ASE with the Sequenom system, i.e. reliable Illumina allelic expression quantification. A cDNA intensity threshold of 11.25 units (average $\log _{2}$ fluorescence) was chosen; below this value, ASE correlation was noted to be weaker (Figure 1). The 576 SNPs with average cDNA intensities above the threshold on the Illumina arrays are listed in Additional file 1: Supplemental Table S3.

Illumina array sensitivity for ASE detection

To assess the capacity of the Illumina BeadArray ${ }^{\mathrm{Tm}}$ ASE platform to detect ASE, we hybridised varying propor-

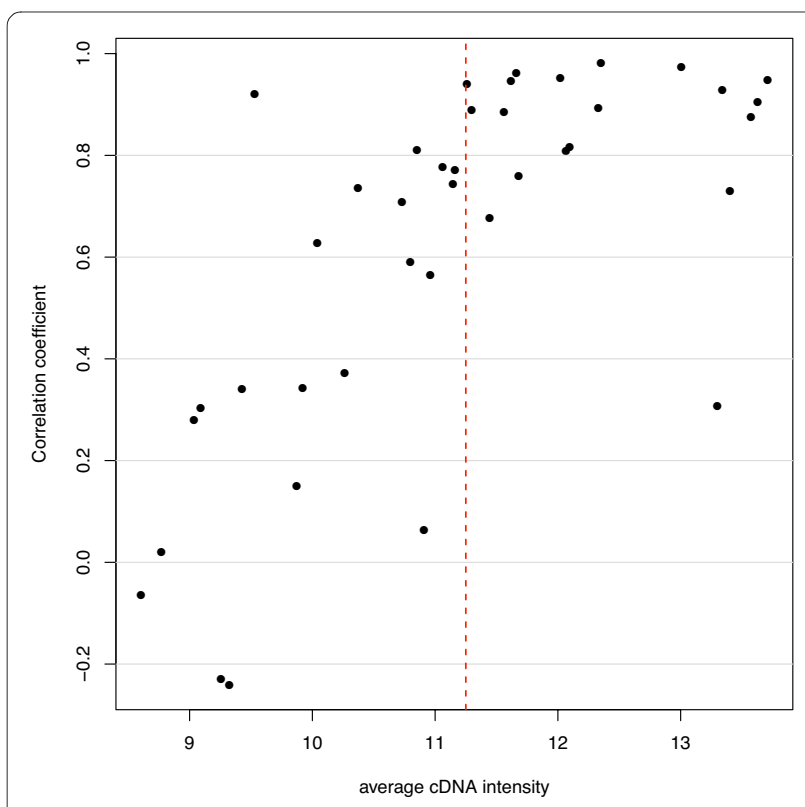

Figure 1 Correlation of Sequenom and Illumina allele quantification. Pearson's correlation coefficient $(r)$ is calculated for the allele-specific quantification on the two platforms and plotted against cDNA intensity (average $\log _{2}$ fluorescence for the 23 placentas) on Illumina. The correlation drops when cDNA average intensity is lower than the 11.25 cut-off (dashed red line). 
tions of homozygous and heterozygous DNAs on the array (Figures 2, 3 and Methods). These 'mixture curves' show that this platform performs well to detect imprinting and strong ASE ( $\geq$ 66-34 ratio) (mean 66-34/34-66 area under the ROC curve $\geq 0.81$ ) but less well to detect moderate ASE ( $\leq 60-40$ ratio) (area under the ROC curve $\leq$ 0.77) (Figure 2).

\section{Illumina array sensitivity for imprinting detection}

Having demonstrated the ability of the Illumina array to quantify strong ASE, we analysed the expression pattern of the 18 imprinted control genes present on the array (Table 2). To detect differential allelic expression, we designed a statistical test (ASE test, see Methods). Being an extreme form of ASE, we should detect imprinting easily if the imprinted gene is sufficiently expressed in human term placenta. Eleven imprinted control genes had a mean cDNA intensity $>11.25$ units (average $\log _{2}$ fluorescence). Eight genes - H19 (Figure 4), PEG3, DLK1, PLAGL1, PEG10, MEST, IGF2AS and ZNF331 (Figure 5, see below) - displayed a pattern characteristic of imprinting (parent-of-origin dependant monoallelic expression). One imprinted control gene, GNAS, was tested by two SNPs, rs3730171 and rs8386, which both had hybridisation intensities above 11.25. Only one placenta was heterozygous for each of the GNAS SNPs, and those two different placentas showed biallelic GNAS expression (Table 2). So, as found by others [23], GNAS was not imprinted in human term placenta. For PHLDA2, only one informative trio was available and showed maternal expression as expected (both parents were heterozygous in the other case). IGF2R, was found to be biallelic for 13

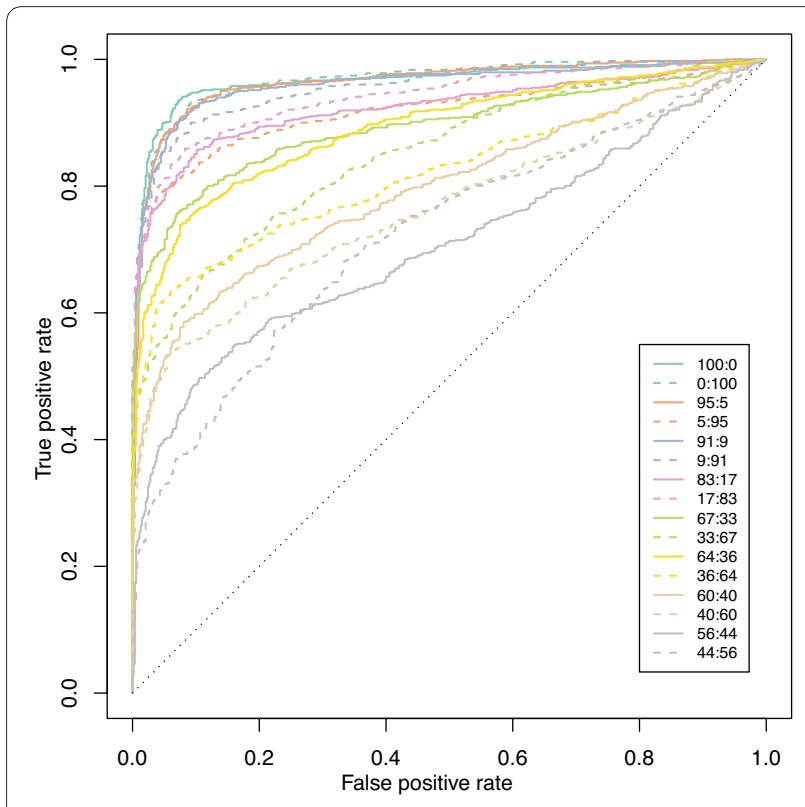

Figure $\mathbf{2}$ ROC plots for the mixture control data set

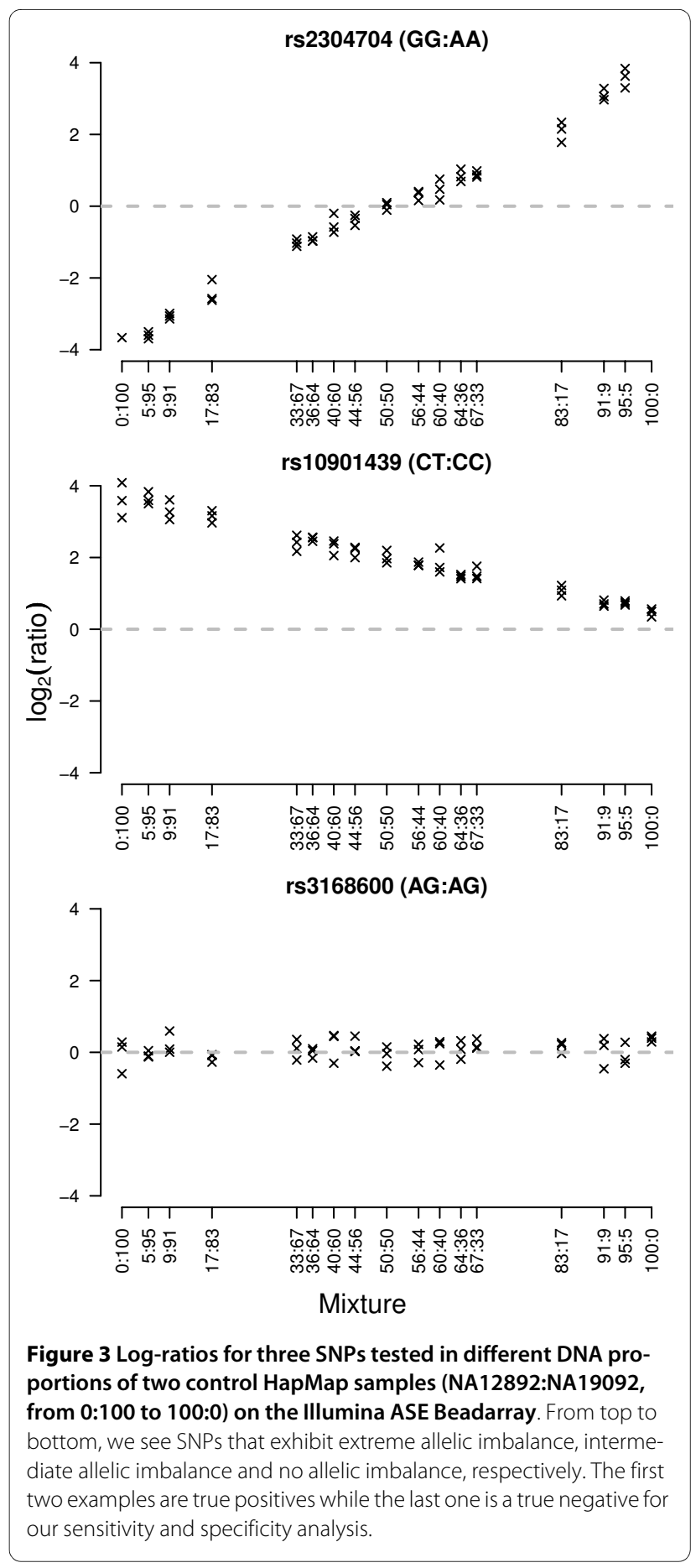

informative samples, as expected in human term placenta [24].

For SNPs of imprinted control genes with intensities $<11.25$, the imprinting pattern became less consistent (Table 2), confirming the value of the threshold determined by the comparison of allelic expression for genes present on both platforms. 


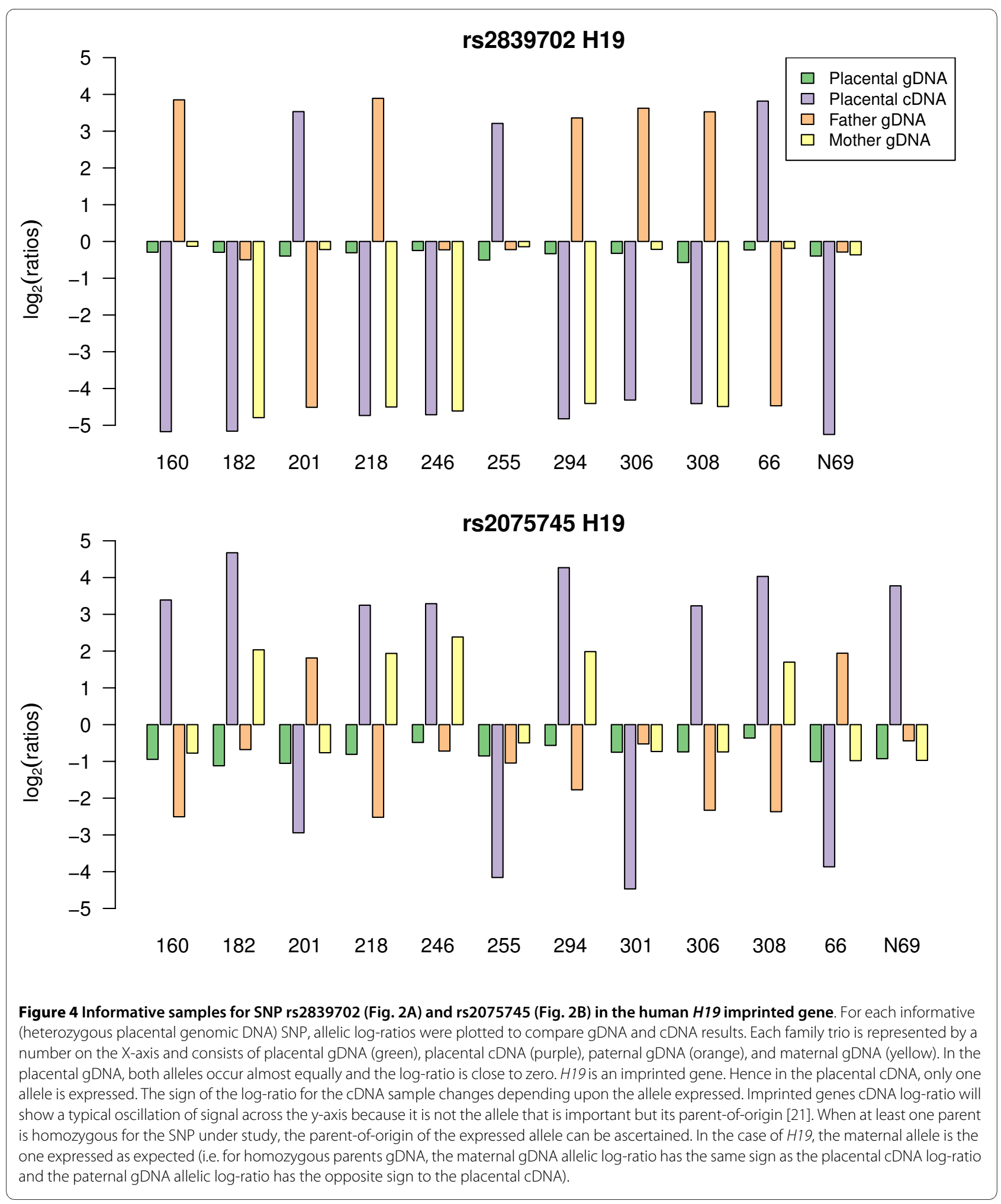

\section{Allele specific expression}

Having established that the Illumina system could detect imprinting and strong allelic expression imbalance, we examined all the genes for evidence of ASE. SNPs were considered to show statistically significant ASE if they satisfied the following criteria: average cDNA intensity across all samples $>11.25$; showed allelic imbalance in expression according to our test (see Methods) in at least $80 \%$ of homozygous cDNA samples; and showed allelic 
Table 2: Imprinted control genes, expression threshold and analysis by our statistical ASE test on the Illumina array.

\begin{tabular}{|c|c|c|c|c|c|c|}
\hline SNP ID & Gene & Chr & $\begin{array}{l}\text { Average } \\
\text { intensity } \\
\text { across all } \\
\text { samples }\end{array}$ & $\begin{array}{l}\text { Total number } \\
\text { of } \\
\text { heterozygous } \\
\text { samples (hets) }\end{array}$ & $\begin{array}{l}\text { Hets showing } \\
\text { ASE: } p<0.01 \\
\& \mid \text { Ifc } \mid>0.585\end{array}$ & $\begin{array}{l}\text { Percentage } \\
\text { of hets } \\
\text { which show } \\
\text { ASE }\end{array}$ \\
\hline rs 2075745 & H19 & 11 & 14.01 & 12 & 12 & $100 \%$ \\
\hline rs1860565 & PEG3 & 19 & 13.63 & 9 & 9 & $100 \%$ \\
\hline rs1802710 & $D L K 1$ & 14 & 13.57 & 15 & 14 & $93 \%$ \\
\hline rs3730171 & GNAS & 20 & 13.54 & 1 & 0 & $0 \%^{+}$ \\
\hline rs2839702 & H19 & 11 & 13.48 & 11 & 11 & $100 \%$ \\
\hline rs998075 & $I G F 2 R$ & 6 & 12.78 & 13 & 0 & $0 \% *$ \\
\hline rs8100247 & ZNF331 & 19 & 12.61 & 13 & 12 & $92 \%$ \\
\hline rs9373409 & PLAGL 1 & 6 & 12.48 & 11 & 9 & $82 \%$ \\
\hline rs13390 & PHLDA2 & 11 & 12.33 & 2 & 2 & $100 \%$ \\
\hline rs10863 & MEST & 7 & 12.32 & 4 & 4 & $100 \%$ \\
\hline rs12982082 & ZNF331 & 19 & 12.27 & 12 & 10 & $83 \%$ \\
\hline rs13073 & PEG10 & 7 & 12.09 & 11 & 10 & $91 \%$ \\
\hline rs8386 & GNAS & 20 & 11.97 & 1 & 0 & $0 \%^{\dagger}$ \\
\hline rs1055359 & PEG3 & 19 & 11.8 & 10 & 10 & $100 \%$ \\
\hline rs1003483 & IGF2AS & 11 & 11.32 & 8 & 7 & $88 \%$ \\
\hline rs854541 & PPP1R9A & 7 & 11.2 & 13 & 0 & $0 \%$ \\
\hline rs2285185 & $\angle 3 M B T L$ & 20 & 10.97 & 10 & 1 & $10 \%$ \\
\hline rs2171492 & CPA4 & 7 & 10.57 & 12 & 0 & $0 \%$ \\
\hline rs2071970 & $\angle 3 M B T L$ & 20 & 10.51 & 11 & 0 & $0 \%$ \\
\hline rs854524 & PPP1R9A & 7 & 10.41 & 10 & 0 & $0 \%$ \\
\hline rs8234 & KCNQ1 & 11 & 10.18 & 10 & 0 & $0 \%$ \\
\hline rs1049846 & PLAGL1 & 6 & 10.06 & 13 & 3 & $23 \%$ \\
\hline rs1800504 & GRB10 & 7 & 9.98 & 14 & 0 & $0 \%$ \\
\hline rs3741208 & IGF2AS & 11 & 9.67 & 10 & 0 & $0 \%$ \\
\hline rs367035 & $S L C 22 A 18$ & 11 & 9.09 & 13 & 0 & $0 \%$ \\
\hline rs3816800 & ATP10A & 15 & 9.09 & 15 & 0 & $0 \%$ \\
\hline rs1570070 & IGF2R & 6 & 9.06 & 0 & 0 & $0 \%$ \\
\hline rs1800900 & GNAS & 20 & 8.86 & 9 & 2 & $22 \%$ \\
\hline rs2066710 & ATP10A & 15 & 8.8 & 16 & 0 & $0 \%$ \\
\hline
\end{tabular}

Imprinted genes tested on the array are listed alongside the exonic SNP used and the intensity obtained for the cDNA (average log ${ }_{2}$ fluorescence for the 23 placentas). The fore last column shows the number of heterozygous samples that are significant for ASE ( $p<0.01)$ and that have a good probe hybridisation signal on the array (absolute log-fold change (Ifc) $>0.58$ ). Examination of the last column (percentage of heterozygous placentas which exhibit statistically significant ASE) shows the difference for reliable detection of imprinting above or below the 11.25 average intensity threshold. ${ }^{*}$ IGF2R is known to be biallelically expressed in human term placenta. ${ }^{+}$The two heterozygous GNAS placentas showed biallelic expression.

imbalance in expression according to our test (see Methods) in at least two heterozygous cDNA samples.

576 out of 1536 SNPs on the array passed the 11.25 intensity threshold indicating sufficient expression in the term placenta for reliable ASE detection (Table 3 and Additional file 1: Supplemental Table 3 for full list). Of these 576 SNPs, 497 (86\%) were polymorphic in our population for at least two individuals and so were informative for the detection of ASE. 261 SNPs passed the additional signal-based quality control criteria (see Methods and Table 3). Using our statistical test, ASE was detected in 56 out of these 261 SNPs. Of these, 44 SNPs 
Table 3: Summary of the DNA and cDNA genotyping for the Illumina assay.

\begin{tabular}{|c|c|c|c|}
\hline & Description & Genes & SNPs \\
\hline A & Tested on the array & 932 & 1536 \\
\hline B & $\begin{array}{l}\text { Above intensity threshold } \\
(11.25)\end{array}$ & 446 & 576 \\
\hline C & $\begin{array}{l}\text { As in B with at least two } \\
\text { heterozygous samples }\end{array}$ & 393 & 497 \\
\hline D & $\begin{array}{l}\text { As in } C \text { with good quality } \\
\text { probe hybridisation in } \\
\text { homozygotes }\end{array}$ & 214 & 261 \\
\hline$E$ & $\begin{array}{l}\text { As in } D \text { with at least two } \\
\text { heterozygotes significant for } \\
\text { ASE }(p<0.01)\end{array}$ & 49 & 56 \\
\hline $\mathrm{F}$ & $\begin{array}{l}\text { As in E for the candidate genes } \\
\text { only }\end{array}$ & 39 (18.2\%) & 44 (16.9\%) \\
\hline
\end{tabular}

targeted 39 candidate genes and 12 SNPs targeted nine control imprinted genes (Table 3).

Five different types of ASE were looked for in the 44 SNPs targeting 39 genes: (1) imprinted, monoallelic expression in a parent-of-origin dependent manner; (2) ASE in a parent-of-origin manner, also called partial imprinting; (3) preferential ASE, where the same allele is expressed at higher levels in each heterozygote whatever its parent-of-origin; (4) random monoallelic expression, where one of the two alleles is completely silenced in a random way; (5) random ASE, where different alleles are expressed at higher levels in different heterozygotes without parental bias (Table 4). To determine which of these patterns of allelic imbalance in expression was detected, log-ratios of informative family-trios were plotted as described in Figure 4 and subjectively categorised (Additional file 3). The patterns of allelic imbalance identified for the 56 SNPs are reported in Table 4.

For the genes exhibiting a statistically significant ASE effect, an imprinting ASE pattern was found for all control imprinted genes and ZNF331 (encoding a zinc finger protein on chromosome 19q13.41, RefSeq NM_018555). Using two SNPs on the Illumina system, rs8100247 (exon 1, 5'UTR) and rs12982082 (exon 2, 5'UTR), ZNF331 showed a consistent pattern of maternal origin for the expressed allele (Figure 5). These results strongly suggest that the ZNF331 transcripts targeted by the SNPs present on the array are imprinted and maternally expressed in the human term placenta. RT-PCR amplification and Sanger sequencing of SNPs in two exons of the ZNF331 transcript (exon 1, 5'UTR and exon 7, CDS) confirmed the maternal expression seen with the Illumina method (Additional file 4).
ZNF331 is thus imprinted in human term placenta. Usually differentially methylated CpG islands are necessary to achieve imprinting. The four 'promoter' $\mathrm{CpG}$ islands (Figure 6) that we could find at the 5 ' extremity of each isoform of ZNF331 were tested for differential methylation. We have been able to amplify $3 \mathrm{CpG}$ islands in bisulphite-treated human term placental DNA. The CpG 100 (promoter of ZNF331 second longest isoform) showed a typical DMR pattern (amplicons are either fully methylated or unmethylated). Unfortunately, no SNP was present in the amplified regions to determine the parental specific methylation of the DMR.

As imprinted genes are often found in clusters, we analysed the CpG island closest to ZNF331 for differential methylation (Figure 7). We found this CpG (located between the DPRX gene and the C19MC miRNA cluster and called CPG 86) to show a typical DMR pattern. Again, no SNP was available to test its parent-specific methylation. So these data suggests that ZNF331 could be part of a new imprinted locus with (at least) two DMRs.

The second imprinted candidate, based on our Illumina array ASE test, is PHACTR2 (phosphatase and actin regulator 2 gene). The PHACTR2 gene contains the SNP rs1082, located in the 3'UTR of the gene, and 10 of 14 informative placentas exhibited ASE dependent on the parent-of-origin of the allele (Figure 8). The fact that the cDNA log-ratio is always smaller than the one seen for homozygous gDNA suggests partial imprinting. Parental genotyping shows that it is always the maternal allele that is more highly expressed.

Partial imprinting of PHACTR2, was confirmed using Sanger sequencing on fourteen placental samples. A recurrent maternal bias was seen between gDNA and 
Table 4: The ASE pattern of genes reaching statistical significance (Illumina Assay).

\begin{tabular}{|c|c|c|c|c|c|c|c|c|}
\hline rsID & Name & Chr & $\begin{array}{l}\text { Imprinting } \\
\text { status }\end{array}$ & $\begin{array}{l}\text { Average } \\
\text { intensity } \\
>11.25\end{array}$ & $\begin{array}{l}\text { Number of } \\
\text { hets }\end{array}$ & $\begin{array}{l}\text { Number of } \\
\text { hets with } \\
p<0.01\end{array}$ & Pattern of ASE & Alleles \\
\hline rs1802710 & $D L K 1$ & 14 & control & 13.57 & 15 & 14 & imprinting & \\
\hline rs8100247 & ZNF331 & 19 & control & 12.61 & 13 & 12 & imprinting & \\
\hline rs2075745 & H19 & 11 & control & 14.01 & 12 & 12 & imprinting & \\
\hline rs2839702 & H19 & 11 & control & 13.48 & 11 & 11 & imprinting & \\
\hline rs1082 & PHACTR2 & 6 & candidate & 12.09 & 14 & 10 & $\begin{array}{l}\text { partial } \\
\text { imprinting }\end{array}$ & \\
\hline rs12982082 & ZNF331 & 19 & control & 12.27 & 12 & 10 & imprinting & \\
\hline rs13073 & PEG10 & 7 & control & 12.09 & 11 & 10 & imprinting & \\
\hline rs1055359 & PEG3 & 19 & control & 11.8 & 10 & 10 & imprinting & \\
\hline rs9373409 & PLAGL1 & 6 & control & 12.48 & 11 & 9 & imprinting & \\
\hline rs1860565 & PEG3 & 19 & control & 13.63 & 9 & 9 & imprinting & \\
\hline rs2309428 & $T J P 2$ & 9 & candidate & 12.86 & 9 & 8 & random ASE & \\
\hline rs178077 & SNAP29 & 22 & candidate & 11.95 & 10 & 7 & random ASE & \\
\hline rs1003483 & IGF2AS & 11 & control & 11.32 & 8 & 7 & imprinting & \\
\hline rs8585 & UBE2V1 & 20 & candidate & 12.36 & 13 & 6 & preferential & $A>G$ \\
\hline rs1130663 & $C D 151$ & 11 & candidate & 12.44 & 18 & 5 & random ASE & \\
\hline rs4664114 & FMNL2 & 2 & candidate & 11.51 & 14 & 5 & random ASE & \\
\hline rs4944960 & XRRA1 & 11 & candidate & 12.43 & 12 & 5 & preferential & $\mathrm{G}>\mathrm{C}$ \\
\hline rs2282336 & $T J P 2$ & 9 & candidate & 12.32 & 9 & 5 & random ASE & \\
\hline rs6633 & $C D K 2 A P 1$ & 12 & candidate & 11.34 & 8 & 5 & random ASE & \\
\hline rs4614 & VPS11 & 11 & candidate & 13.09 & 13 & 4 & random ASE & \\
\hline rs3817672 & TFRC & 3 & candidate & 12.07 & 13 & 4 & * preferential & $\mathrm{T}>\mathrm{C}$ \\
\hline rs2905 & C14orf130 & 14 & candidate & 11.33 & 12 & 4 & random ASE & \\
\hline rs12190287 & TCF21 & 6 & candidate & 12.88 & 10 & 4 & random ASE & \\
\hline rs3809865 & ITGB3 & 17 & candidate & 12.09 & 10 & 4 & random ASE & \\
\hline rs10863 & MEST & 7 & control & 12.32 & 4 & 4 & imprinting & \\
\hline rs915894 & $\mathrm{NOTCH} 4$ & 6 & candidate & 13.44 & 16 & 3 & * preferential & $A>C$ \\
\hline rs754615 & CAST & 5 & candidate & 13.71 & 14 & 3 & preferential & $\mathrm{G}>\mathrm{C}$ \\
\hline rs838896 & SCARB1 & 12 & candidate & 12.62 & 10 & 3 & random ASE & \\
\hline rs5758651 & TCF20 & 22 & candidate & 12.4 & 10 & 3 & random ASE & \\
\hline rs11699879 & NCOA3 & 20 & candidate & 12.68 & 9 & 3 & random ASE & \\
\hline rs838891 & SCARB1 & 12 & candidate & 11.85 & 9 & 3 & random ASE & \\
\hline rs2425009 & MYH7B & 20 & candidate & 11.68 & 9 & 3 & random ASE & \\
\hline rs9749449 & ZNF211 & 19 & candidate & 11.71 & 6 & 3 & random ASE & \\
\hline rs4797 & SQSTM1 & 5 & candidate & 14.48 & 18 & 2 & preferential & $\mathrm{G}>\mathrm{A}$ \\
\hline rs1128933 & MAN2C1 & 15 & candidate & 12.71 & 16 & 2 & preferential & $C>T$ \\
\hline rs10277 & SQSTM1 & 5 & candidate & 11.78 & 16 & 2 & preferential & $\mathrm{G}>\mathrm{A}$ \\
\hline rs2249057 & NM_006031 & 21 & candidate & 13.31 & 13 & 2 & * preferential & $\mathrm{C}>\mathrm{A}$ \\
\hline rs7226091 & MGC16597 & 17 & candidate & 11.83 & 13 & 2 & * preferential & $C>G$ \\
\hline rs1043618 & HSPA1A & 6 & candidate & 13.6 & 12 & 2 & random ASE & \\
\hline rs17085249 & ELL2 & 5 & candidate & 12.98 & 12 & 2 & random ASE & \\
\hline rs2255255 & CRNKL1 & 20 & candidate & 12.86 & 12 & 2 & random ASE & \\
\hline
\end{tabular}


Table 4: The ASE pattern of genes reaching statistical significance (Illumina Assay). (Continued)

\begin{tabular}{|c|c|c|c|c|c|c|c|c|}
\hline rs2013162 & IRF6 & 1 & candidate & 12.64 & 12 & 2 & * preferential & $C>A$ \\
\hline rs11121567 & $P G D$ & 1 & candidate & 12.03 & 12 & 2 & * preferential & $A>G$ \\
\hline rs3780473 & ACO1 & 9 & candidate & 11.67 & 12 & 2 & random ASE & \\
\hline rs4669 & $T G F B I$ & 5 & candidate & 13.27 & 11 & 2 & random ASE & \\
\hline rs7242 & SERPINE1 & 7 & candidate & 13.01 & 11 & 2 & random ASE & \\
\hline rs2788478 & FLJ10300 & 7 & candidate & 13.04 & 10 & 2 & random ASE & \\
\hline rs2271108 & DOCK5 & 8 & candidate & 12.85 & 10 & 2 & random ASE & \\
\hline rs552282 & PPFIA1 & 11 & candidate & 11.58 & 10 & 2 & * preferential & $C>T$ \\
\hline rs1044116 & NOTCH3 & 19 & candidate & 12.21 & 9 & 2 & random ASE & \\
\hline rs7204628 & MGC24665 & 16 & candidate & 11.84 & 9 & 2 & random ASE & \\
\hline rs844 & FCGR2B & 1 & candidate & 13.05 & 8 & 2 & * preferential & $C>T$ \\
\hline rs11156878 & KIAA0391 & 14 & candidate & 12.15 & 5 & 2 & random ASE & \\
\hline rs 12780 & PRDM8 & 4 & candidate & 12.02 & 5 & 2 & random ASE & \\
\hline rs5919 & ITGB3 & 17 & candidate & 11.55 & 4 & 2 & random ASE & \\
\hline rs13390 & PHLDA2 & 11 & control & 12.33 & 2 & 2 & imprinting & \\
\hline
\end{tabular}

All SNPs had an average intensity above 11.25 and all or a subset of heterozygous samples had a significant statistical ASE test $(p<0.01)$. For each SNP, the ASE pattern (see text for details) was subjectively determined by examination of the bar charts, designed as in Figure 4, for all heterozygous samples. In case of preferential expression, the allele that was more expressed is indicated in the last column. ${ }^{*}$ For these SNPs, the preferential bias is weaker.

cDNA sequence traces overlapping the same PHACTR2 3'UTR SNP (rs1082) (Figure 9). These sequencing results confirm the partial imprinting of PHACTR2 in human term placenta and the ability of the Illumina BeadArray ${ }^{\text {mm }}$ platform to detect ASE.

To examine further the strength of allelic silencing observed in our data for all imprinted genes (i.e. complete to partial imprinting), raw allelic values, averaged over all cDNAs from informative individuals, were plotted for the imprinted control genes and the most significant imprinted candidate gene on the array (Figure 10). The difference of expression between the two alleles of a known imprinted gene varies from a 23 -fold difference (PEG3-rs1860565) to a 6.4-fold difference (DLK1rs1802710). For ZNF331, the difference is 5-fold for rs12982082 and 11-fold for rs8100247, and for the partially imprinted gene PHACTR2, 2.6-fold (Figure 10). These results show that the repression of the silenced allele is not complete for all control imprinted genes and that there is a continuum from 'complete imprinting' to 'partial imprinting'. While our results could suggest that it is likely that most or all 'completely imprinted' genes have already been found in the placenta (see discussion), our PHACTR2 study indicates that partially imprinted genes could have been labelled as 'biallelic' and that several other partially imprinted genes could still be found and characterised.
Of the 56 SNPs (49 genes) statistically significant with our ASE test, 12 SNPs were located in nine of our selected imprinted control genes (DLK1, H19, IGF2AS, MEST, PEG3, PEG10, PLAGL1, PHLDA2, ZNF331) and one SNP was localised in PHACTR2 and its ASE pattern was compatible with partial imprinting (see above).

Of the 43 remaining SNPs (39 genes), six (five genes) showed an allelic preferential pattern when visually examined (UBE2V1, XRRA1, CAST, SQSTM1, MAN2C1; see Additional file 5) and eight showed possible allelic preference (Table 4 and Additional file 3). The others were too variable to be assigned a precise ASE pattern and could correspond to random allelic bias, epistatic allelic preferential expression, bipolar ASE (see Discussion) [25] or false positives.

To investigate these 43 significant ASE SNPs further, we used the Genevar Database (T-P. Yang and E. Dermitzakis, manuscript in preparation) to check for cis-effects for the same 43 SNPs and 39 genes in LCLs from eight HapMap3 populations (CEU, CHB, JPT, GIH, MKK, YRI, LWK, MEX) (B. Stranger and E. Dermitzakis, manuscript in preparation). The database allows searching for a specific SNP-gene pair showing an expression quantitative trait locus (eQTL), for cis-eQTLs arising from a specific SNP or for cis-eQTL SNPs acting on a specific gene $[19,20]$. In other words, using this database, we can look for the effect of a specific SNP on the transcription of a specific gene (SNP-gene pair eQTL), the effect of a spe- 
cific SNP on all (tested) genes located in the vicinity of this SNP (SNP cis-eQTL) or we can examine the effect on the transcription level of a specific gene by any SNPs located in the vicinity of this gene (gene cis-eQTL). We can also examine transcription level of a specific gene by any tested SNPs in the vicinity of the gene (cis-effect) or far away from the gene (trans-effect). We found respectively nine, four and two of these types of eQTLs in the database corresponding to our ASE SNPs and genes. This suggests that 15 of our 43 ASE SNPs (35\%) could be genuine examples of allelic preferential expression in two different human tissues, namely term placenta and LCLs [26]. Five of the $15 \mathrm{eQTL}$ were found to overlap with the six ASE significant SNPs-genes pairs showing a prominent allelic preferential bias (see Additional file 5): four SNP-gene pair eQTLs (SQSTM1-rs10277, - rs 4797; MAN2C1-rs1128933; CAST-rs754615) and one gene ciseQTL (XRRA1 (rs4944960 does not exist in Genevar)). $U B E 2 V 1$ showed only a marginal gene-eQTL effect while rs8585 was also not in the Genevar database. So all four SNP-gene pairs tested in both tissues and four of the five (80\%) genes showing significant preferential allelic bias in placenta also showed a strong preferential allelic bias in LCLs. In addition to the validation of our placental experiments, this overlap strongly suggests that our most significant preferential allelic biases (Additional file 5) are genuine (and probably ubiquitous).

\section{Discussion}

Our data demonstrate that quantitative genotyping technologies like the Sequenom Mass Spectrometer and Illumina Beadarray ${ }^{\text {tm }}$ platforms are reliable in the detection of strong allelic skewing as shown by the correct identification of known imprinted genes and different patterns of ASE from the data. We have found that allelic imbalances in expression are common in the candidates we analysed in the human term placenta and that true monoallelic expression (imprinted or random) is a rare phenomenon. We found only one new 'partially imprinted' gene $(0.5 \%)$, while ASE was present in $18 \%$ of the candidate genes passing our quality control criteria. Such levels of ASE are similar to the results seen in cell lines or other somatic tissues [12-15,21].

Our data show that ZNF331 is imprinted in human term placenta and expressed from the maternal allele. ZNF331 (also known as ZNF463) was first shown to exhibit monoallelic expression in a parent-of-origin manner in lymphoblastoid cell lines [13,21], although the parent-of-origin orientation of ZNF331 in these studies was not clear (paternal in one study, maternal in the other). No obvious explanation would easily explain this discrepancy. It would be interesting to study ZNF331 allelic mode of expression in a range of human tissues and in an isoform-specific manner.
In addition, our methylation results (Figures 6 and 7) suggest that ZNF331 could be part of a new imprinted locus with (at least) two DMRs. Recently, Tsai and colleagues showed the same DMR pattern for the CpG 86 (the one located between $D P R X$ and $C 19 M C$ genes) independently suggesting that the 'ZNF331-C19MC' locus could be a new imprinted locus [27]. C19MC seems to be mainly expressed in placenta and fetal brain [28-30], a pattern that would perfectly suit the expression of an imprinted gene. Finally, ZNF331 and C19MC seem to be primate specific genes (no murine orthologue for ZNF331 was found using Ensembl or UCSC; and C19MC is primate-specific [28-30]). This probably explains why this locus was not found in previous mouse genome wide screens for imprinted loci. Hence, all aggregated results suggest a possible importance of the ZNF331-C19MC locus in human placental-fetal growth, metabolism and cancer. Being primate specific genes, the determination of their functional role in development will be a challenge.

We found PHACTR2 to be partially imprinted in placenta (Figures 8 and 9). PHACTR2 is located on chromosome 6q24.2, $114 \mathrm{~kb}$ from PLAGL1 a known imprinted gene (previously called $Z A C 1$ ). Loss of imprinting of PLAGL1 is seen in transient neonatal diabetes [31,32]. PHACTR2 is a member of a family of four actin and protein phosphatase 1 (PP1) binding proteins highly expressed in the brain [33,34]. The function of PHACTR2 in placenta is unknown. PHACTR1, 3 and 4 have roles in brain and neural tube development and in cell spreading $[35,36]$. Mouse strain allele specific dominant expression has been shown in brain for an isoform of Phactr3 (i.e., only the Phactr3 NMRI allele of exon $1 \mathrm{C}$ is expressed in NMRI/Cast heterozygous F1 progeny whatever the parent-of-origin of the NMRI allele) [37]. So, our results show that PHACTR2 is partially imprinted in placenta, and, with other work, suggest that the PHACTR gene family could be prone to complex epigenetic regulation.

In total across the two platforms, we experimentally studied 183 genes identified as candidates for imprinted expression by prior bioinformatics approaches $[8,9]$. Luedi et al. [8] predicted 600 genes to be imprinted out of 23,788 murine autosomal annotated genes. We have tested 155 of these 600 mouse candidates and found one that exhibited (partial) imprinting in the term placenta. In another study of these murine candidates [38], one (KCNK9) out of 16 genes selected from the 600 candidates was found to be imprinted in the mouse and human brain. Some of the 16 candidates tested by Ruf et al. [39] were selected due to their proximity to known imprinted genes. In our results the one gene that exhibited partial imprinting, PHACTR2 is located adjacent to PLAGL1, a known imprinted gene (previously called ZAC1). Combined with the prior observations that imprinted genes 

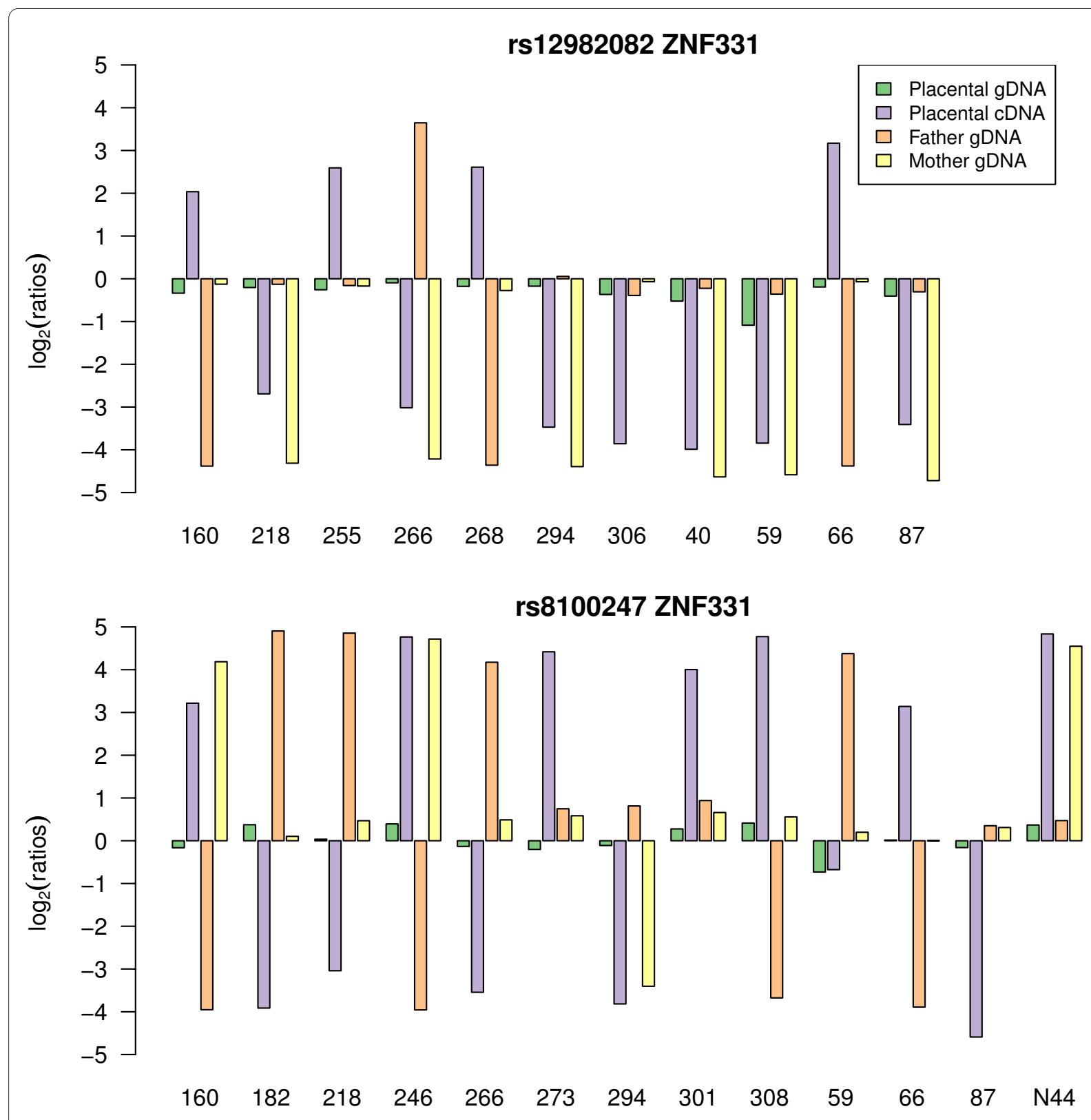

Figure 5 Monoallelic maternal expression of ZNF331 as detected by two SNPs rs12982082 and rs8100247. Bar chart designed as in Figure 4.

often occur in clusters, these data suggest that if there are more imprinted genes to be found they may lie close to other imprinted genes.

Recently, Luedi and colleagues generated a list of 156 candidate human imprinted genes [40]. Given that nearly all genes that are imprinted in human are also imprinted in the mouse, it is surprising that the mouse and human prediction lists overlap for only a few candidates. Noncoding features like repeats were used to predict candidates and it is possible that there were differences in the assembly quality of these features in the versions of the human (Ensembl version 20) and mouse (Ensembl version 16$)$ genomes used for these studies $[8,40]$. It would be interesting to test the algorithms on the most recent assemblies of both genomes. None of the 28 candidates identified by mining EST databases [9] that we tested was imprinted in placenta. Thus, only one of the 183 candidates predicted by bioinformatics methods that we tested was found (partially) imprinted in placenta. The poor specificity of the bioinformatics predictions in placenta raises two possibilities: either, the bioinformatics predictions have low specificity overall and only a handful 


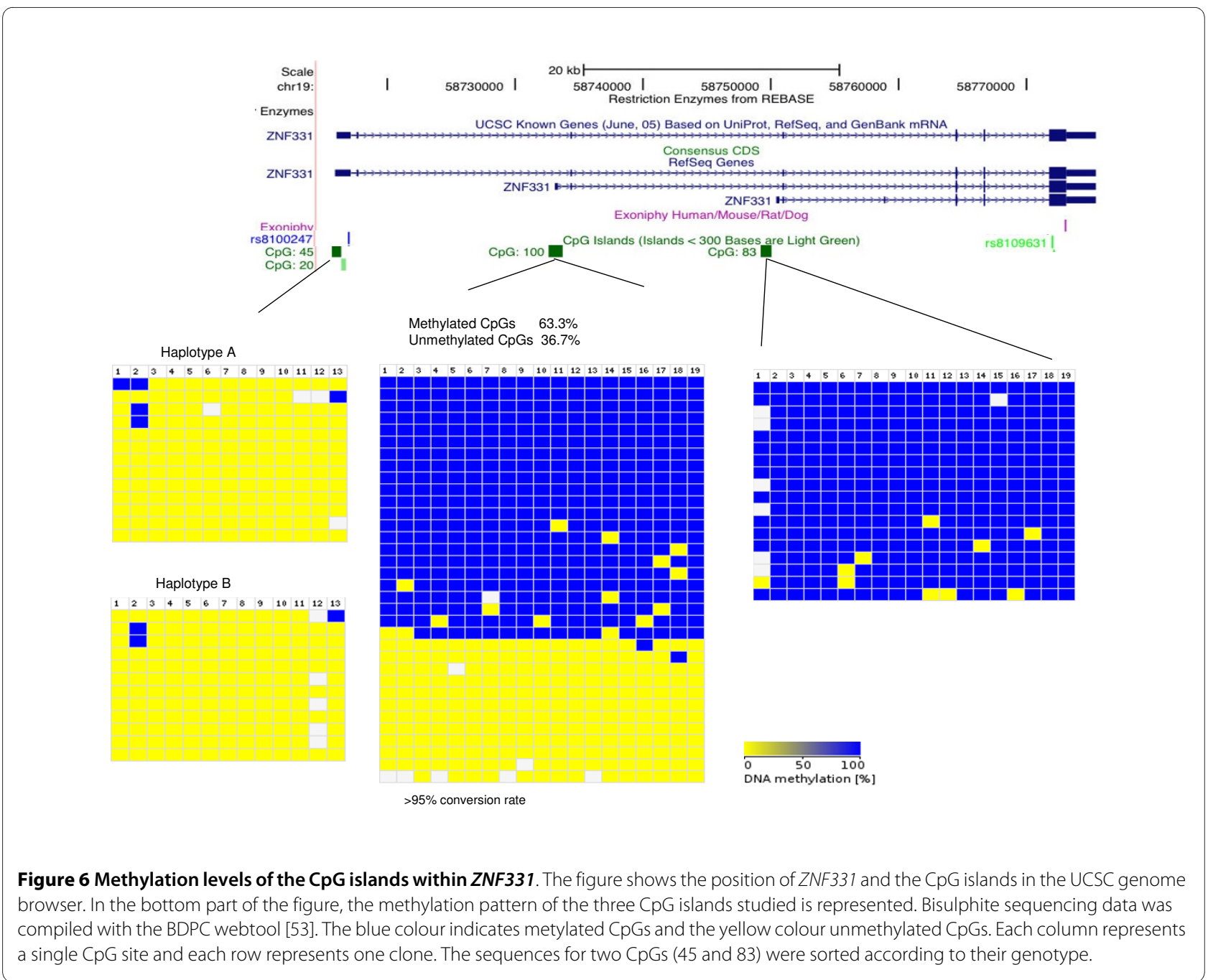

imprinted genes are still to be discovered or the predictions are correctly identifying imprinting in tissues other than placenta. Most phenotypes with a heritability compatible with imprinted gene disruption have been explained [6]. However, new imprinted genes are still being discovered:NLRP2 and OSBPL1A in placenta [15], ZNF331 in placenta (this work) and in LCLs [13,21], KCNK9 in brain [39,40], DLGAP2 in testis [40]. Hence it is possible that new imprinted genes will mainly be discovered in a tissue-specific manner and that more subtle phenotypes could be associated with their disruption.

We analysed five modes of ASE (imprinted, partial imprinting, preferential, monoallelic random, random ASE). Recently, Cheverud and colleagues suggested that different bipolar modes of ASE could exist [25,41]. Bipolar ASE shows allele specific bias depending first on the parent-of-origin of the allele and second on heterozygous or homozygous status for this allele (a mode of allelic expression inheritance that was previously only known in the callipyge sheep [42]). Considering the bipolar associ- ated growth and metabolic phenotypes described by Cheverud et al. in the adult mouse [25], it will be interesting to explore bipolar ASE in human tissues. However, the platforms used in this study would need to test many more trios with more replicates to approach the precision required to investigate such complex ASE patterns.

Our quantitative allelic expression results for the imprinted control genes present on the array showed that the 'silencing' of the repressed allele is not always absolute (Figure 10). It is more of a continuum from complete silencing (e.g. PEG3, H19, and MEST) to partial silencing (e.g., DLK1, IGF2AS and PHACTR2). These results agree with the recent work of Lambertini et al, who showed some expression of the 'silenced' allele in human term placenta [23]. For example, for DLK1 such incomplete silencing was present for several individuals on both the Illumina and Sequenom platforms. We also documented one placenta showing nearly 50-50 biallelic expression of DLK1 (data not shown). Sakatani and colleagues have already described such complete relaxation of imprinting 


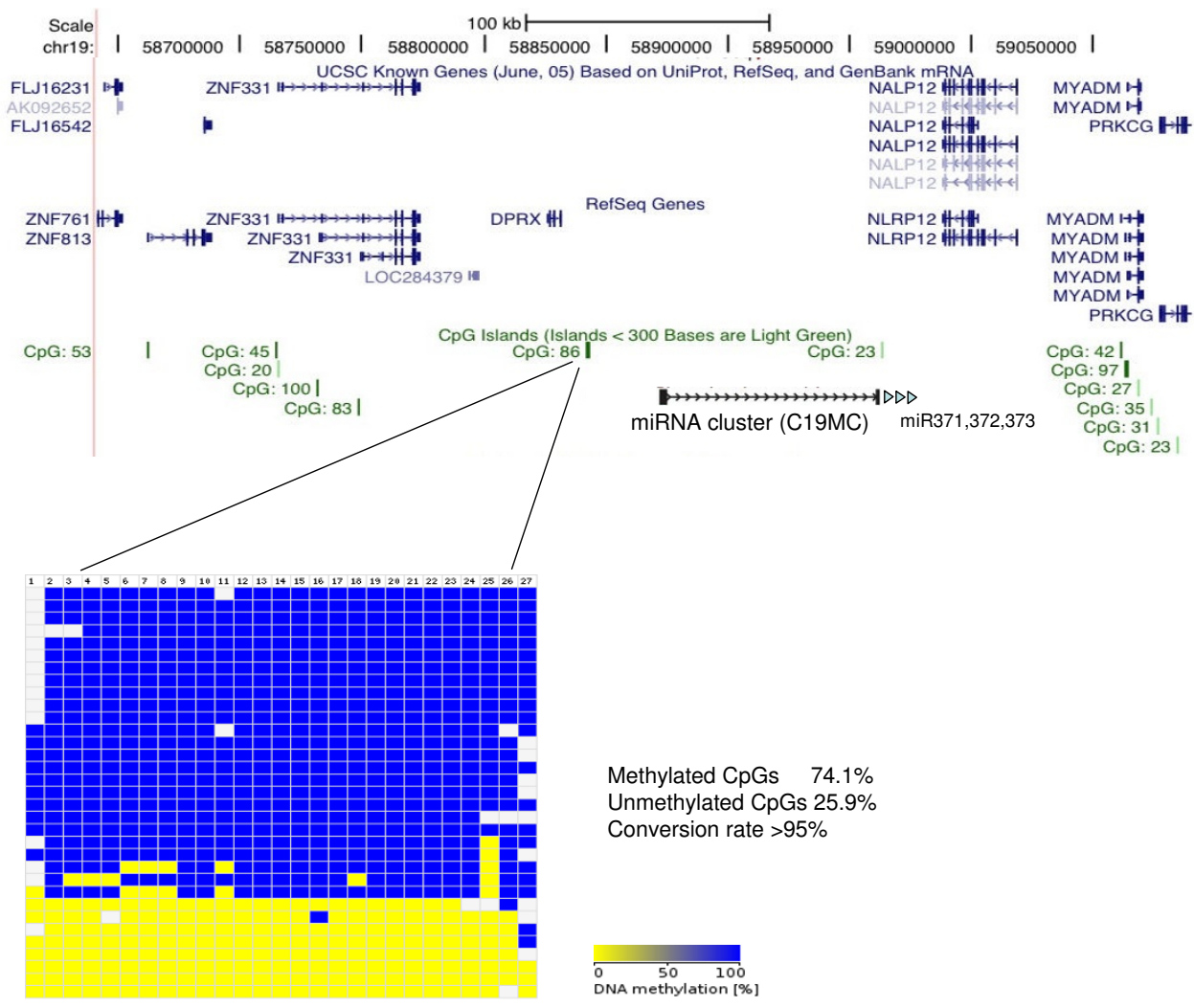

Figure 7 Methylation levels of CpG86 in ZNF331 locus. The figure, generated with UCSC genome browser, indicates the position of the CpG island in relation to the location of ZNF331, its neighbouring genes and miRNAs. Bisulphite sequencing data was compiled with the BDPC webtool as in Figure 6. No SNP was present in the amplified sequence.

for IGF2 [43]. As them, we also found one term placenta (10\%) showing biallelic expression for IGF2 (data not shown). The pathological importance of such loss of imprinting in a 'healthy' human term placenta is not known. Hence, our quantitative allelic expression in imprinted genes suggest that term placenta can rarely show complete loss of imprinting for IGF2 and DLK1, that parent-specific allelic expression is a continuum from complete silencing of one parental allele to a parentally biased expression of the two alleles, and that some partially imprinted genes could still be found.

\section{Conclusion}

Both Sequenom MassArray and Illumina GoldenGate platforms were sensitive enough to study imprinting and strong ASE (= 66-34 ratio). Four patterns of ASE (imprinting, partial imprinting, preferential ASE, and random ASE) were found in human term placenta. Prior bioinformatics predictions were not useful to identify new imprinted genes in the human term placenta, suggesting that screening of other tissues and/or refinement of prediction methods may be necessary. We showed that ZNF331, a known lymphoblastoid cell imprinted gene, is maternally expressed in human term placenta. The possibility that ZNF331 is ubiquitously imprinted argues for further study of its function in metabolism, behaviour, fetal development and cancer. We showed that two potential DMRs are present in the primate-specific ZNF331-C19MC locus. We showed that PHACTR2, a neighbour of the imprinted gene PLAGL1, is partially imprinted in human placenta, the maternal allele being more highly expressed. Such a result calls for further evaluation of the allelic expression landscape of the complex and gene-rich human PHACTR2-PLAGL1 locus. Demonstration of incomplete silencing of the repressed allele for several control imprinted genes and PHACTR2 indicates that partially imprinted genes can be identified with appropriate screening tools. On the Illumina array, 39 candidate genes were statistically significant for our ASE test (18\% of the candidate genes passing quality controls). Finally, our results suggest that ASE is a common 


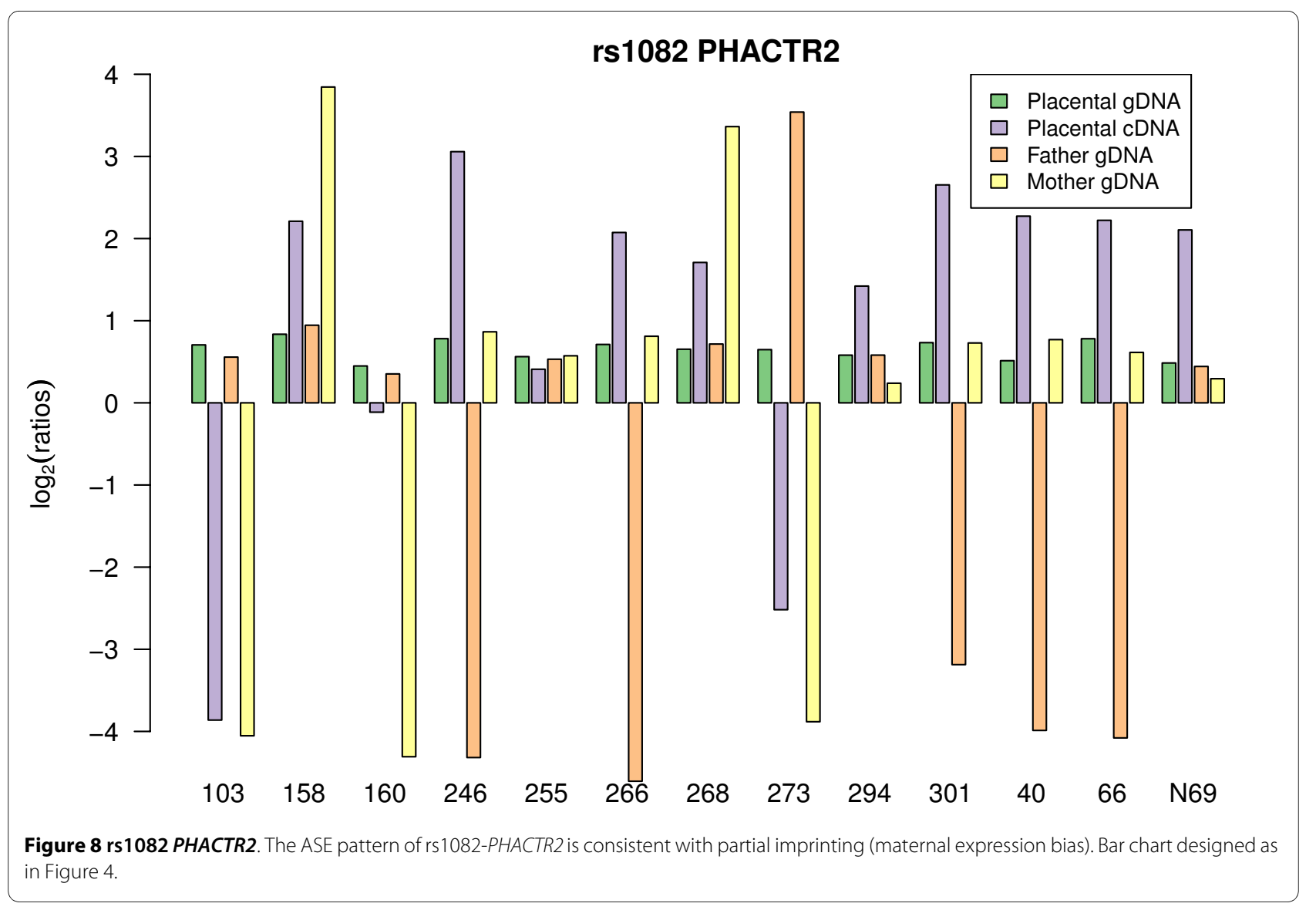

variability factor in placental tissue and should be thoroughly studied in normal and pathological pregnancy.

\section{Methods}

\section{DNA and RNA preparation}

Placental trio samples consisting of placental tissues with corresponding maternal and paternal blood samples were collected from consenting pregnant mothers of European ancestry at Queen Charlotte's and Chelsea Hospital (local ethics approval 2001/6029). Samples were washed in sterile PBS and snap frozen in liquid nitrogen. A set of 24 trios was randomly chosen from the tissue bank. For one trio, the genotyping of parental DNAs revealed it was not a biological family and parental information was removed from subsequent analyses. Genomic DNA (gDNA) was extracted from placental tissue samples and peripheral blood using standard phenol-chloroform separation. Total RNA was extracted from homogenised placental tissues using Trizol (Invitrogen). RNA was treated with Turbo DNA-free (Ambion) to minimize genomic DNA contamination, concentrated and further cleaned with RNeasy MinElute columns (Qiagen). Total RNA and gDNA were quantified using a spectrophotometer and

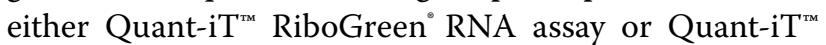
PicoGreen DNA assay (Invitrogen). For the Sequenom platform, single stranded cDNA was synthesised from 250 ng of RNA with Superscript III reverse-transcriptase (RT) (Invitrogen) and random hexamers. Duplicate sets of samples were processed with RT omitted to detect genomic contamination of the RNA. Both sets were diluted at 1/50 before being assayed. For the Illumina platform, double stranded cDNA was synthesised from $250 \mathrm{ng}$ of total RNA. The first strand was synthesised with Superscript ${ }^{\mathrm{ma}}$ III RT (Invitrogen) and randoms hexamers. The second strand was synthesised with DNA polymerase I (Invitrogen) and ribonuclease H (Invitrogen). The 96-well plates containing the double-stranded cDNA samples were cleaned using Multiscreen ${ }^{\circ} \mathrm{PCR}_{\mu 96}$ filtration plates (Millipore) before being assayed on the Illumina ASE array.

\section{Sequenom Assay}

Control and candidate genes were selected for quantitative genotyping using the homogeneous MassEXTEND (hME) assay (Sequenom, Inc.) according to their expression levels in placenta in the Unigene database http:// www.ncbi.nlm.nih.gov/UniGene. The SNPs chosen were located in the 5'UTR, 3'UTR, or exons and had a minor allelic frequency (MAF) $>0.15$ in our population of European ancestry (dbSNP Build ID: 125 and 126, http:// 


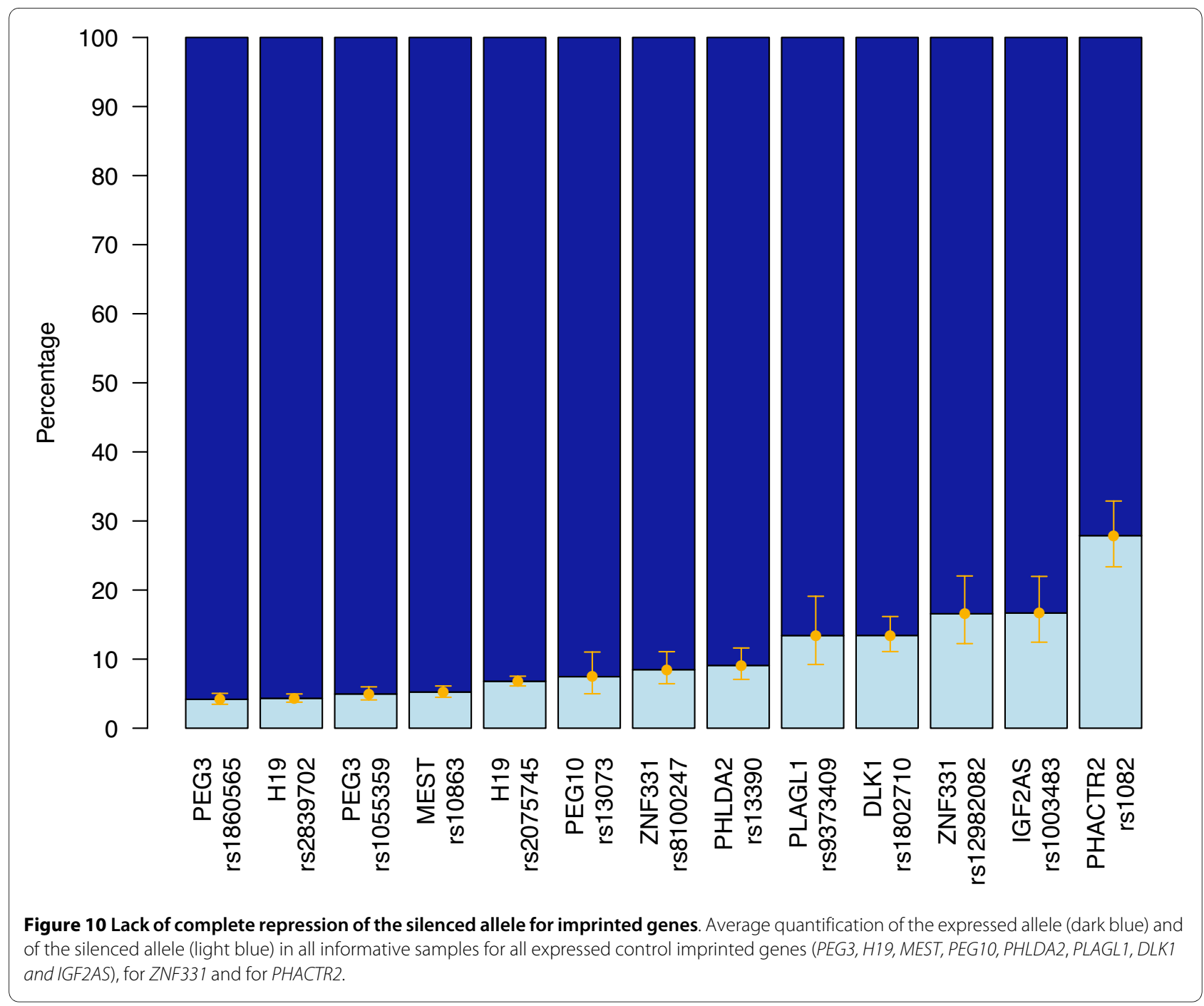

www.ncbi.nlm.nih.gov/SNP/. One SNP per gene was studied for seven biallelic controls, six human imprinted genes, seven orthologues of mouse imprinted genes, 26 human candidates [9], and 100 orthologues of mouse candidate imprinted genes [8] (Additional file 1: Supplemental Table S1).

The MassArray system (Sequenom, Inc.) consists of a primer extension assay for genotyping and quantitation of alleles by MALDI-TOF (matrix-assisted laser desorption/ionization time-of-flight) mass spectrometry [44]. Three different primers (two for amplification and one allele-specific MassEXTEND primer) were designed for each targeted SNP using SpectroDesigner (Sequenom, Inc.) within the exon or the UTRs. PCR amplification was followed by shrimp alkaline phosphatase (SAP) treatment. The primer extension reaction generates different mass signals for the two alleles. SNPs were multiplexed in threes according to the termination mix used. Samples were purified using SpectroCLEAN (Sequenom). Sam- ples were then spotted on the chip (SpectroCHIP, Sequenom) with the MassArray nanodispenser and analysed by SpectroREADER mass spectrometer (Sequenom). Genotypes were called by the proprietary software (SpectroTyper v2.0). Primer sequences and thermocycling conditions are available upon request.

\section{Sequenom analysis}

To find new imprinted genes or ASE, genotype calls were filtered to include only the genotypes that had been called with the "conservative" rating. The percentage of genotyping assays called in this way for each SNP was referred to as the success rate (SR) and was calculated for gDNA and cDNA. The ratio of cDNA to gDNA SR was used to filter out lowly expressed genes. Genotyping with a SR ratio $\geq 75 \%$ was taken forward in the analysis. Calls were then filtered to select trios with heterozygous placental genomic DNA. On these trios, a one-tailed paired $t$-test was used, for each SNP, to compare allelic quantification of the two alleles in placental cDNA and in placental 
genomic DNA. $P$-values were then adjusted using the Benjamini-Hochberg method to control false discovery rate [45]. The analysis was carried out in $\mathrm{R}$ [46].

\section{Illumina Assay}

The oligo pool of 1536 SNPs of the GoldenGate ASE Array (Illumina, Inc., USA) included 18 known imprinted genes, four housekeeping genes, 11 genes shown to be preferentially expressed in the placenta [22], ten genes predicted to be imprinted in humans [9], ten orthologues of mouse imprinted genes, 35 genes that are differentially expressed according to infant weight [22], six polycomb genes and 124 human orthologues of genes predicted to be imprinted in mouse [8]; all of which were selected based on their placental expression in the Unigene database http://www.ncbi.nlm.nih.gov/UniGene (see Additional file 1: Supplemental Table S2 for a list of SNPs and genes). All SNPs chosen were located within the exons or UTRs of the targeted genes in order to be present in the spliced mRNA. SNPs with the highest minor allele frequency (MAF) in our population in the single nucleotide polymorphisms database (dbSNP Build ID: 125 and 126), http://www.ncbi.nlm.nih.gov/SNP/ and best Illumina design scores in our candidate genes were preferred. Alleles were differentiated by $\mathrm{Cy} 3$ and $\mathrm{Cy} 5$ labelled probes [47].

Paired gDNA (250 ng) and double-stranded cDNA (made from $250 \mathrm{ng}$ total RNA, see above) were identically processed and hybridised to a standard 96-sample Sentrix Array Matrix according to the manufacturer's instructions for GoldenGate genotyping assays (Illumina, Inc., USA) [48]. After hybridisation for 16 hours, arrays were scanned with a Bead Station (Illumina, Inc., USA). For each placental sample, gDNA and cDNA were assayed on the same plate, and the whole plate analysis was replicated on a different day. For two cDNA samples replicates, cDNA amplification was not obtained. Parental gDNA genotyping was performed on a separate plate (not replicated). The genotypes were called using Illumina's proprietary software (BeadStudio and GenCall) with the gDNA signals as input. The composition of the trios was imported so that Mendelian errors could be highlighted during the manual curation of the genotyping. Arrays with a low dynamic range were discarded and repeated. The raw data from this experiment is available in the ArrayExpress database http://www.ebi.ac.uk/arrayexpress under accession number E-TABM-796.

\section{Illumina Data Analysis}

The raw $\mathrm{Cy} 3$ and $\mathrm{Cy} 5$ intensities from all beads on an array were quantile normalised between channels. Logratios $\left(\log _{2}(\mathrm{Cy} 5 / \mathrm{Cy} 3)\right)$ and average log-intensities (1/ $\left.2 \log _{2}(\mathrm{Cy} 5 \times \mathrm{Cy} 3)\right)$ were calculated for each bead on each array. Outliers greater than 3 mean absolute deviations
(MADs) from the median of each bead type were removed as per Illumina's standard method and the remaining values were averaged to obtain a summary logratio and average log-intensity for each bead type (i.e., mean of $\sim 30$ beads per SNP) on each array. The summarized data were normalised per array by median centering the log-ratios to have median zero.

To test for ASE, we used the following method. Linear models were fitted to the cDNA log-ratios to summarise the replicate observations. After empirical Bayes shrinkage of the SNP-wise variances, moderated $t$-statistics were calculated [49]. Raw $p$-values from these $t$-tests were adjusted globally for multiple testing using the method of Benjamini and Hochberg to control the false discovery rate [45]. Our criteria for ASE required that SNPs satisfy the following conditions: (1) average intensity across all samples greater than 11.25 (Illumina arbitrary fluorescence units); (2) at least 2 heterozygotes (based on BeadStudio calls from gDNA samples) with adjusted $p$-values less than 0.01 and absolute log-foldchanges greater than 0.585 and (3) at least $80 \%$ of homozygotes with adjusted $p$-values less than 0.01 and absolute log-fold-changes greater than 0.585 . The intensity cut-off was based on the concordance between Illumina and Sequenom data, with probes expressed below this level less reliably quantified on the Illumina arrays (Figure 1). The log-fold-change cut-off of 0.585 was based on the mixture data (Figure 2). This experiment showed that true positives were more difficult to detect on the Illumina arrays in mixtures at or below 60:40/40:60 (equivalent to absolute log-ratios less than $\log _{2}(60 / 40)=$ 0.585 ). The homozygote criteria (3) ensured that the two alleles could be reliably distinguished in the cDNA samples. All analyses were carried out in $\mathrm{R}$ using the beadarray [50] and limma packages [51].

\section{Mixture Analysis}

For the control experiment, gDNA mixtures of two HapMap individuals (NA12892:NA19092) (Coriell, Camden, New Jersey, United States) were created in the following proportions: $0 \%: 100 \%, \quad 5 \%: 95 \%, \quad 91 \%: 9 \%, 83 \%: 17 \%$, 67\%:33\%, 64\%:36\%, 60\%:40\%, 56\%:44\%， 50\%:50\%, 44\%:56\%, 40\%:60\%, 36\%:64\%, 33\%:67\%, 17\%:83\%, 9\%:91\%, 5\%:95\% and $100 \%: 0 \%$. Each mixture was hybridized in duplicate using the same experimental protocol. Data were preprocessed and normalised as described in the previous section.

A linear model was fitted to each SNP as described previously, and contrasts were obtained to give all pairwise comparisons between a given mixture and the 50\%:50\% mixture. This corrects for dye biases and systematic shifts which are present for SNPs which are either heterozygous and homozygous (i.e. $\mathrm{AA}: \mathrm{AB}, \mathrm{BB}: \mathrm{AB}, \mathrm{AB}: \mathrm{AA}$ or $\mathrm{AB}: \mathrm{BB}$ ) or have the same genotype (AA:AA, $\mathrm{BB}: \mathrm{BB}$ or $\mathrm{AB}: \mathrm{AB})$ in 
the two individuals. Moderated $t$-statistics were calculated using the empirical Bayes shrinkage procedure [49] to test the null hypothesis that each contrast was equal to 0 (i.e. no allelic imbalance). Sensitivity and specificity calculations were made for each contrast by ranking SNPs by their log-odds and using a priori genotype information on which SNPs are true positives/negatives for allelic imbalance.

Genotypes for NA12892 and NA19092 were downloaded from HapMart http://hapmart.hapmap.org/ BioMart/martview, version 21, NCBI Build 35) for the SNPs on the array. SNPs with known allelic imbalances between these individuals (782), such as those which are either homozygous and different (AA:BB or BB:AA), or heterozygous and homozygous (AA:AB, BB:AB, $\mathrm{AB}: \mathrm{AA}$ or $A B: B B)$, form the true positive set. SNPs which have the same genotype for each individual (AA:AA, BB:BB or $A B: A B)$ should not change with mixing concentration, and comprise the true negative set (533). SNPs with missing data ( 15 with $\mathrm{NN}$ calls) and those with IDs that could not be found in HapMart (206) were excluded from the analysis.

\section{Platform Correlation}

Pearson correlation coefficients were calculated for 38 SNPs using log-ratios from samples assayed using both the Illumina arrays and Sequenom assay (log-ratios calculated as $\log _{2}[($ seque_x +1$) /($ seque_y +1$\left.)]\right)$.

\section{Sanger sequencing}

Using Primer3 http://frodo.wi.mit.edu/, one set of primers was designed to be used for both PCR and RT-PCR. Primer sequences and thermocycling conditions are available upon request. PCR and RT-PCR products were cleaned with Microclean (Microzone) and sequenced using standard ABI sequencing technology (Big Dye v1.1).

\section{Methylation study}

Bisulphite converted gDNA samples were prepared and cleaned using the EZ DNA methylation-Gold ${ }^{\text {mu }}$ kit (Zymo, $\mathrm{CA}$ ) according to the manufacturer's instructions. For each CpG island of interest, bisulphite primers were designed using the MethPrimer webtool http://www.urogene.org/methprimer/index1.html[52]. Hotstar Taq polymerase (Qiagen, West Sussex, UK) was used for 45 PCR cycles to amplify converted gDNA samples. One to three $\mu \mathrm{l}$ of crude PCR product was ligated into $\mathrm{PGEM}^{\circ}-\mathrm{T}^{\circ} \mathrm{Vec}-$ tor System (Promega) as per manufacturer's instructions. Ligations were then incubated at $4^{\circ} \mathrm{C}$ with JM109 high efficiency competent bacterial cells (Promega) for 30 minutes. The bacterial cells were then heat shocked at $42^{\circ} \mathrm{C}$ for 45 seconds in a pre-heated water bath and immediately returned on ice for 2 minutes. White colo- nies were selected for sequencing and resuspended in 100 $\mu \mathrm{l}$ of LB-broth. The resuspended colonies were incubated at $37^{\circ} \mathrm{C}$ for 1 to 2 hours. Two $\mu \mathrm{l}$ of each colony was amplified by standard PCR reaction with M13 forward and reverse primers or the specific primers designed for the $\mathrm{CpG}$ island of interest. Sequences were analysed to determine bisulphite conversion of $\mathrm{CpG}$ sites using Bisulphite Sequencing DNA Methylation Analysis (BISMA) webtool http://biochem.jacobs-university.de/BDPC/BISMA/ index.php[53].

\section{Additional material}

\section{Additional file 1 Supplemental Table S1: List of SNPs and genes}

tested on Sequenom platform. Supplemental Table S2: List of SNPs and genes tested on Illumina platform. Supplemental Table S3: SNPs with an average intensity $>11.25$ units

Additional file 2 Figure showing preferential allelic expression of ACSS2 on the Sequenom platform. Averaged allelic ratios for heterozygous gDNA and $c D N A$ were plotted. The higher $C / T$ ratio in $C D N A$ shows preferential $\mathrm{C}$ allele expression (t-test $\mathrm{p}$ value $=0.0075$ ).

Additional file 3 Figure confirming imprinting of ZNF331 in human term placenta by Sanger Sequencing. Sequences (top for rs 8100247 (exon 1, 5'UTR) and bottom for rs8109631 (exon 7, CDS)) of informative term placenta samples in gDNA and CDNA with corresponding genotyping data for the father and the mother. Complete imprinting is visible for the exon 1 SNP, while partial imprinting is present for the exon 7 SNP suggesting an isoform specific imprinting. It is the maternal allele that is (more) expressed.

Additional file 4 Figure showing statistically significant genes exhibiting preferential ASE on the Illumina array. ASE for SQSTM1, UBE2V1 and XRRA1 is evident while the effect for CAST and MAN2C1 is more subtle.

\section{Authors' contributions}

Conceived and designed the experiments: CD, GS, GEM, ID. Performed the experiments: CD, MSF. Analysed the data: CD, MER, GS, IMS, TC, ST, GEM, ID. Provided reagents and materials: SAA, SC, PS, DK, PD, ETD, GEM, ID. Drafted the paper: CD, MER, GS. Reviewed the paper: PS, ST, GEM, ID. All authors read and approved the final manuscript.

\section{Acknowledgements}

We thank all patients who donated samples and Sophia Apostolidou for sample collection. We thank all members of the Genotyping Facility Team at the Sanger Institute for their expert technical assistance.

Funding: CD is a Wellbeing of Women Fellow. PS and GEM acknowledge funding from the MRC, the Wellcome Trust, Wellbeing of Women, and SPARKS. ST acknowledges support from Cancer Research UK and Hutchison Whampoa Limited. DK, PD, ETD and ID acknowledge funding from the Wellcome Trust.

\section{Author Details}

'Wellcome Trust Sanger Institute, Hinxton, Cambridge, CB10 1 SA, UK, ${ }^{2}$ Molecular and Clinical Genetics Unit, Institute of Child Health, London, WC1 1EH, UK, ${ }^{3}$ Department of Obstetrics and Gynecology, Institute for Women's Health, University College London, London, WC1E 6HX, UK, ${ }^{4}$ Department of Oncology, University of Cambridge, CRUK Cambridge Research Institute, Li Ka Shing Centre, Robinson Way, Cambridge, CB2 ORE, UK, ${ }^{5}$ Bioinformatics Division, The Walter and Eliza Hall Institute of Medical Research, Parkville, Victoria 3052, Australia, ${ }^{6}$ Department of Genetic Medicine and Development, University of Geneva Medical School, Geneva, Switzerland and ${ }^{7}$ European Bioinformatics Institute, Hinxton, Cambridge, CB10 1SD, UK

Received: 1 October 2009 Accepted: 19 April 2010 Published: 19 April 2010 


\section{References}

1. Pernis B, Chiappino G, Kelus AS, Gell PG: Cellular localization of immunoglobulins with different allotypic specificities in rabbit lymphoid tissues. J Exp Med 1965, 122(5):853-876.

2. Chess A, Simon I, Cedar H, Axel R: Allelic inactivation regulates olfactory receptor gene expression. Cell 1994, 78(5):823-834.

3. Reik W, Walter J: Genomic imprinting: parental influence on the genome. Nature Reviews Genetics 2001, 2(1):21-32.

4. Tycko B, Morison IM: Physiological functions of imprinted genes. Journal of Cellular Physiology 2002, 192(3):245-258.

5. Feinberg AP: An epigenetic approach to cancer etiology. Cancer $J 2007$, 13(1):70-74

6. Morison IM, Ramsay JP, Spencer HG: A census of mammalian imprinting. Trends in Genetics 2005, 21(8):457-465.

7. Morison IM, Reeve AE: A catalogue of imprinted genes and parent-oforigin effects in humans and animals. Human Molecular Genetics 1998 7(10):1599-1609.

8. Luedi PP, Hartemink AJ, Jirtle RL: Genome-wide prediction of imprinted murine genes. Genome Research 2005, 15(6):875-884.

9. Seoighe C, Nembaware V, Scheffler K: Maximum likelihood inference of imprinting and allele-specific expression from EST data. Bioinformatics 2006, 22(24):3032-3039.

10. Coan PM, Burton GJ, Ferguson-Smith AC: Imprinted genes in the placenta--a review. Placenta 2005, 26(Suppl A):S10-20

11. Yan H, Yuan W, Velculescu VE, Vogelstein B, Kinzler KW: Allelic variation in human gene expression. Science 2002, 297(5584):1143.

12. Lo HS, Wang Z, Hu Y, Yang HH, Gere S, Buetow KH, Lee MP: Allelic variation in gene expression is common in the human genome. Genome Research 2003, 13(8):1855-1862.

13. Pant PV, Tao H, Beilharz EJ, Ballinger DG, Cox DR, Frazer KA: Analysis of allelic differential expression in human white blood cells. Genome Research 2006, 16(3):331-9.

14. Gimelbrant A, Hutchinson JN, Thompson BR, Chess A: Widespread monoallelic expression on human autosomes. Science 2007, 318(5853):1136-1140

15. Bjornsson HT, Albert TJ, Ladd-Acosta CM, Green RD, Rongione MA, Middle CM, Irizarry RA, Broman KW, Feinberg AP: SNP-specific array-based allelespecific expression analysis. Genome Research 2008, 18(5):771-779.

16. Knight JC, Keating BJ, Rockett KA, Kwiatkowski DP: In vivo characterization of regulatory polymorphisms by allele-specific quantification of RNA polymerase loading. Nature Genetics 2003, 33(4):469-475

17. Serre D, Gurd S, Ge B, Sladek R, Sinnett D, Harmsen E, Bibikova M, Chudin E, Barker DL, Dickinson T, et al:: Differential allelic expression in the human genome: a robust approach to identify genetic and epigenetic cis-acting mechanisms regulating gene expression. PLoS Genet 2008, 4(2):e1000006.

18. Plass C, Shibata H, Kalcheva I, Mullins L, Kotelevtseva N, Mullins J, Kato R, Sasaki H, Hirotsune S, Okazaki Y, et al:: Identification of Grf1 on mouse chromosome 9 as an imprinted gene by RLGS-M. Nature Genetics 1996, 14(1):106-109.

19. Stranger BE, Forrest MS, Dunning M, Ingle CE, Beazley C, Thorne N, Redon R, Bird CP, de Grassi A, Lee C, et al:: Relative impact of nucleotide and copy number variation on gene expression phenotypes. Science 2007, 315(5813):848-853

20. Stranger BE, Nica AC, Forrest MS, Dimas A, Bird CP, Beazley C, Ingle CE, Dunning M, Flicek P, Koller D, et al.: Population genomics of human gene expression. Nature Genetics 2007, 39(10):1217-1224.

21. Pollard KS, Serre D, Wang X, Tao H, Grundberg E, Hudson TJ, Clark AG, Frazer K: A genome-wide approach to identifying novel-imprinted genes. Hum Genet 2008, 122(6):625-634.

22. Sood R, Zehnder JL, Druzin ML, Brown PO: Gene expression patterns in human placenta. Proceedings of the National Academy of Sciences of the United States of America 2006, 103(14):5478-5483.

23. Lambertini L, Diplas Al, Lee MJ, Sperling R, Chen J, Wetmur J: A sensitive functional assay reveals frequent loss of genomic imprinting in human placenta. Epigenetics 2008, 3(5):261-269.

24. Monk D, Arnaud P, Apostolidou S, Hills FA, Kelsey G, Stanier P, Feil R, Moore GE: Limited evolutionary conservation of imprinting in the human placenta. Proceedings of the National Academy of Sciences of the United States of America 2006, 103(17):6623-6628.
25. Cheverud JM, Hager R, Roseman C, Fawcett G, Wang B, Wolf JB: Genomic imprinting effects on adult body composition in mice. Proceedings of the National Academy of Sciences of the United States of America 2008 105(11):4253-4258

26. Dimas AS, Deutsch S, Stranger BE, Montgomery SB, Borel C, Attar-Cohen $H$, Ingle C, Beazley C, Arcelus MG, Sekowska M, et al:: Common Regulatory Variation Impacts Gene Expression in a Cell Type-Dependent Manner. Science 2009, 325(5945):1246-50.

27. Tsai KW, Kao HW, Chen HC, Chen SJ, Lin WC: Epigenetic control of the expression of a primate-specific microRNA cluster in human cancer cells. Epigenetics 2009, 4(8):587-592.

28. Bentwich I, Avniel A, Karov Y, Aharonov R, Gilad S, Barad O, Barzilai A, Einat $P$, Einav $U$, Meiri E, et al:: Identification of hundreds of conserved and nonconserved human microRNAs. Nature Genetics 2005, 37(7):766-770

29. Berezikov E, Thuemmler F, van Laake LW, Kondova I, Bontrop R, Cuppen E, Plasterk RH: Diversity of microRNAs in human and chimpanzee brain. Nature Genetics 2006, 38(12):1375-1377.

30. Zhang $R$, Wang $Y Q$, Su B: Molecular evolution of a primate-specific microRNA family. Mol Biol Evol 2008, 25(7):1493-1502.

31. Gardner RJ, Mackay DJ, Mungall AJ, Polychronakos C, Siebert R, Shield JP, Temple IK, Robinson DO: An imprinted locus associated with transient neonatal diabetes mellitus. Hum Mol Genet 2000, 9(4):589-596.

32. Ma D, Shield JP, Dean W, Leclerc I, Knauf C, Burcelin RR, Rutter GA, Kelsey G: Impaired glucose homeostasis in transgenic mice expressing the human transient neonatal diabetes mellitus locus, TNDM. J Clin Invest 2004, 114(3):339-348.

33. Sagara J, Higuchi T, Hattori Y, Moriya M, Sarvotham H, Shima H, Shirato H, Kikuchi K, Taniguchi S: Scapinin, a putative protein phosphatase-1 regulatory subunit associated with the nuclear nonchromatin structure. J Biol Chem 2003, 278(46):45611-45619.

34. Allen PB, Greenfield AT, Svenningsson P, Haspeslagh DC, Greengard P: Phactrs 1-4: A family of protein phosphatase 1 and actin regulatory proteins. Proceedings of the National Academy of Sciences of the United States of America 2004, 101(18):7187-7192.

35. Sagara J, Arata T, Taniguchi S: Scapinin, the protein phosphatase 1 binding protein, enhances cell spreading and motility by interacting with the actin cytoskeleton. PLOS One 2009, 4(1):e4247.

36. Kim TH, Goodman J, Anderson KV, Niswander L: Phactr4 regulates neural tube and optic fissure closure by controlling PP1-, Rb-, and E2F1regulated cell-cycle progression. Dev Cell 2007, 13(1):87-102.

37. Worch S, Hansmann I, Schlote D: Paramutation-like effects at the mouse scapinin (Phactr3) locus. J Mol Biol 2008, 377(3):605-608

38. Ruf N, Bahring S, Galetzka D, Pliushch G, Luft FC, Nurnberg P, Haaf T, Kelsey $\mathrm{G}$, Zechner U: Sequence-based bioinformatic prediction and QUASEP identify genomic imprinting of the KCNK9 potassium channel gene in mouse and human. Hum Mol Genet 2007, 16(21):2591-2599.

39. Ruf N, Bahring S, Galetzka D, Pliushch G, Luft FC, Nurnberg P, Haaf T, Kelsey $G$, Zechner U: Sequence-based bioinformatic prediction and QUASEP identify genomic imprinting of the KCNK9 potassium channel gene in mouse and human. Human Molecular Genetics 2007, 16(21):2591-2599.

40. Luedi PP, Dietrich FS, Weidman JR, Bosko JM, Jirtle RL, Hartemink AJ: Computational and experimental identification of novel human imprinted genes. Genome Res 2007, 17(12):1723-1730.

41. Wolf JB, Cheverud JM, Roseman C, Hager R: Genome-wide analysis reveals a complex pattern of genomic imprinting in mice. PLoS Genet 2008, 4(6):e1000091.

42. Cockett NE, Jackson SP, Shay TL, Farnir F, Berghmans S, Snowder GD, Nielsen DM, Georges M: Polar overdominance at the ovine callipyge locus. Science 1996, 273(5272):236-238.

43. Sakatani T, Wei M, Katoh M, Okita C, Wada D, Mitsuya K, Meguro M, Ikeguchi M, Ito H, Tycko B, et al:: Epigenetic heterogeneity at imprinted loci in normal populations. Biochemical and Biophysical Research Communications 2001, 283(5):1124-1130.

44. Stanssens P, Zabeau M, Meersseman G, Remes G, Gansemans Y, Storm N Hartmer R, Honisch C, Rodi CP, Bocker S, et al:: High-throughput MALDITOF discovery of genomic sequence polymorphisms. Genome Research 2004, 14(1):126-133.

45. Benjamini $Y$, Hochberg $Y$ : Controlling the false discovery rate: a practical and powerful approach to multiple testing. Journal of the Royal Statistical Society Series B (Methodological) 1995:289-300. 
46. R Development Core Team: R: A Language and Environment for Statistical Computing 2009 [http://www.R-project.org]. R Foundation for Statistical Computing, Vienna, Austria

47. Gunderson KL, Steemers FJ, Lee G, Mendoza LG, Chee MS: A genomewide scalable SNP genotyping assay using microarray technology. Nature Genetics 2005, 37(5):549-554.

48. Fan JB, Gunderson KL, Bibikova M, Yeakley JM, Chen J, Wickham Garcia E, Lebruska LL, Laurent M, Shen R, Barker D: Illumina universal bead arrays. Methods Enzymo/ 2006, 410:57-73.

49. Smyth GK: Linear models and empirical bayes methods for assessing differential expression in microarray experiments. Statistical Applications in Genetics and Molecular Biology 2004, 3:Article3.

50. Dunning MJ, Smith ML, Ritchie ME, Tavaré S: beadarray: R classes and methods for Illumina bead-based data. Bioinformatics 2007 23(16):2183-2184.

51. Limma [http://bioinf.wehi.edu.au/limma/

52. Li LC, Dahiya R: MethPrimer: designing primers for methylation PCRs. Bioinformatics 2002, 18(11):1427-1431

53. Rohde C, Zhang Y, Jurkowski TP, Stamerjohanns $H$, Reinhardt R, Jeltsch A: Bisulfite sequencing Data Presentation and Compilation (BDPC) web server--a useful tool for DNA methylation analysis. Nucleic Acids Res 2008, 36(5):e34.

doi: 10.1186/1471-2156-11-25

Cite this article as: Daelemans et al., High-throughput analysis of candidate imprinted genes and allele-specific gene expression in the human term placenta BMC Genetics 2010, 11:25

Submit your next manuscript to BioMed Central and take full advantage of:

- Convenient online submission

- Thorough peer review

- No space constraints or color figure charges

- Immediate publication on acceptance

- Inclusion in PubMed, CAS, Scopus and Google Scholar

- Research which is freely available for redistribution

Submit your manuscript at www.biomedcentral.com/submit
C) Biomed Central 